\title{
Using a mobile health application to support self-management in COPD:
}

\author{
a qualitative study
}

\begin{abstract}
Background

Telehealth shows promise for supporting patients in managing their long-term health conditions, such as chronic obstructive pulmonary disease (COPD). However, it is currently unclear how patients, and particularly older people, may benefit from these technological interventions.

\section{Aim}

To explore patients' expectations and experiences of using a mobile telehealth-based (mHealth) application and to determine how such a system may impact on their perceived wellbeing and ability to manage their COPD.

\section{Design and setting}

Embedded qualitative study using interviews with patients with COPD from various community NHS services: respiratory community nursing service, general practice, and pulmonary rehabilitation.
\end{abstract}

\section{Method}

An embedded qualitative study was conducted to which patients were recruited using purposive sampling to achieve maximum variation. Interviews were carried out prior to receiving the $\mathrm{mHealth}$ system and again after a 6-month period. Data were analysed using a grounded theory approach.

\section{Results}

The sample comprised 19 patients laged 50-85 years) with varied levels of computer skills. Patients identified no difficulties in using the mHealth application. The main themes encapsulating patients' experience of using the mHealth application related to an increased awareness of the variability of their symptoms (onset of exacerbation and recovery time) and reassurance through monitoring (continuity of care).

\section{Conclusion}

Patients were able to use the mHealth application, interpret clinical data, and use these within their self-management approach regardless of previous knowledge. Telehealth interventions can complement current clinical care pathways to support self-management behaviour.

\section{Keywords}

chronic obstructive pulmonary disease; longterm conditions; mobile health application; qualitative research; respiratory conditions self-management; telehealth.

\section{INTRODUCTION}

Chronic obstructive pulmonary disease (COPD) accounts for an increasing burden on both the individual $1^{1,2}$ and the NHS, with one in eight emergency admissions relating to COPD and one in three patients with COPD being readmitted within 28 days of a hospital admission for an exacerbation. ${ }^{3}$

Effective self-management of COPD, including the early recognition and treatment of exacerbations, has the potential to improve outcomes such as the patient's quality of life and to reduce hospital admissions.4.5 Telehealth is increasingly seen as a way to bridge the gap between professional care and patient self-management. ${ }^{6}$ A Cochrane review of telehealth for COPD carried out in 2011 found that such interventions have potential for a positive impact on patients' quality of life and hospitalisation rates, but that further research is needed to understand how telehealth contributes to this because of the complexity of the interventions.? Previous qualitative research on the patient experience of COPD telehealth interventions has found mixed perceptions. Patients perceived interventions to have both positive (symptom awareness and early detection of exacerbations), ${ }^{8}$ and negative aspects lanxiety of misinterpretations of clinical data, and threats to self-identity, through reminders of ageing, illness and

V Williams, BSc, PhD, qualitative researcher; A Farmer, MRCGP, FRCGP, DM, professor of general practice, Department of Primary Care Health Sciences; L Tarassenko, MA, DPhil, professor or engineering and head of department, Department of Engineering Science, Institute of Biomedical Engineering, University of Oxford, Oxford. J Price, DPhil, MRCPsych, clinical tutor in psychiatry, Department of Psychiatry, Warneford Hospital, University of Oxford, Oxford. M Hardinge, MBChB, MD, consultant respiratory physician, Oxford Centre of Respiratory Medicine, Oxford University Hospitals NHS Trust, Churchill Hospital, Oxford. dependence). ${ }^{10}$ Patients also expressed concerns over their relationship with healthcare professionals, which may be threatened by the introduction of telehealth. ${ }^{11}$

The embedded qualitative study presented here was part of a pilot study to refine the COPD telehealth intervention, preceding a randomised controlled trial. ${ }^{12}$ The qualitative study aimed to explore patients' expectations and experiences of using a mobile (mHealth) application to support self-management of COPD.

\section{The mHealth application}

The EDGE (sElf-management anD support proGrammE) COPD project aims to evaluate the efficacy of a multi-component mHealth intervention, delivered via a tablet computer, to improve quality of life in patients with COPD. ${ }^{12}$ This mHealth intervention focused on simplicity of use (touch screen, no keyboard), and consisted of a symptom diary (Figure 1),13 remote self-monitoring (pulse oximetry) (Figure 2), and multimedia educational and self-management materials (videos, text, and images).

Patients were asked to complete the symptom diary and pulse oximetry daily to understand individual variability. These data were reviewed at regular intervals (but not daily) by a respiratory research nurse, but patients were aware that they would have

\section{Address for correspondence}

Veronika Williams, Nuffield Department of Primary Care Health Sciences, University of Oxford, Gibson Building, Radcliffe Observatory Quarter, Woodstock Road, Oxford, OX2 6GG.

E-mail: veronika.williamsaphc.ox.ac.uk

Submitted: 10 December 2013; Editor's response: 1 February 2014; final acceptance: 11 March 2014 (CBritish Journal of General Practice This is the full-length article (published online 30 Jun 2014) of an abridged version published in print. Cite this article as: Br J Gen Pract 2014; DOI: 10.3399/bjgp14X680473. 


\section{How this fits in \\ Telehealth has increasingly received attention as a way to bridge the gap between professional health care and patient self-management. Previous research has explored the patient perspective of telehealth in managing chronic obstructive pulmonary disease (COPD) and has reported mixed results in terms of perceived benefits and disadvantages. The findings of this study show that a mobile health intervention in COPD can be used by patients regardless of their previous computer experience, gives a sense of continuity of care, and promotes self-management behaviour as perceived by patients.}

Figure 1. mHealth interface: initial screen display on tablet computer.

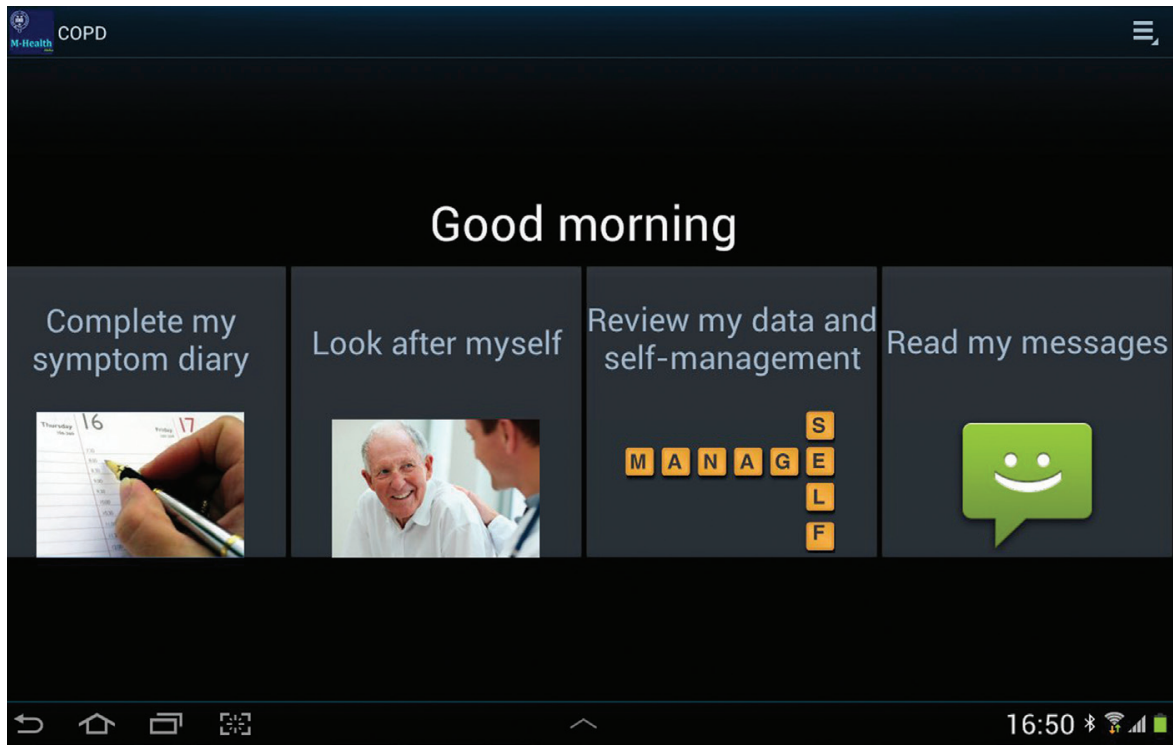

Figure 2. Pulse oximetry: pulse rate and oxygen saturation values are automatically sent via Bluetooth to the tablet computer.

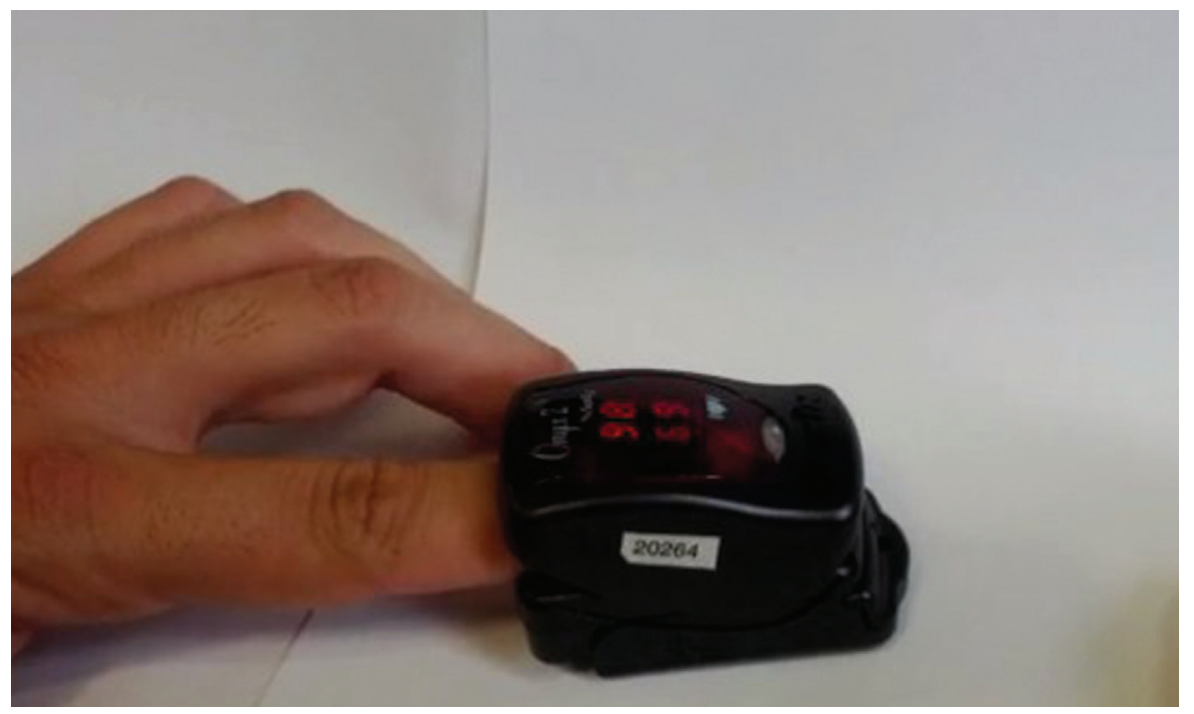

to contact their usual healthcare providers in case of emergencies. Other information available to patients included educational leaflets on smoking cessation, diet, and breathing techniques, as well as video information on the correct use of inhalers.

Minimal training on how to use the application was given to patients at the outset of the study, and the application included integrated training and advice on how to use the pulse oximeter.

\section{METHOD}

\section{Data collection}

Patients meeting the eligibility criteria (Box 1) were identified and recruited from respiratory community records, pulmonary rehabilitation programmes, and GP practices by a research nurse, with the aim of recruiting a maximum variation sample ICOPD severity and length of diagnosis, and sociodemographic background), thus including a wide range of patient experiences.

Data were collected through individual interviews (see Appendices 1 and 2 for a list of interview questions), which were carried out in patients' homes. The first interview prior to using the mHealth intervention focused on how COPD impacted on the patient's life and how they currently managed their COPD. The second interview was carried out after a 6-month period and focused on how their use of the mHealth intervention impacted on their self-management experience. Interviews lasted between 35 and 90 minutes. Interview questions were revisited after each interview to explore issues identified through constant comparative analysis in subsequent interviews. Field notes were recorded immediately after the interview to provide context of the interview and aid the analytic process.

Although the qualitative researcher was part of the EDGE COPD project team, data collection and analysis for this interview study was carried out separately from the intervention development and quantitative pilot study.

\section{Analysis}

The interview guide included questions relating to participants' expectations, views, and experiences of using the mHealth intervention. Interviews were audiorecorded and transcribed verbatim, and anonymised transcripts were imported into NVivo 10 (qualitative software data program) to facilitate organisation and analysis of data. Analysis followed a grounded theory approach $^{14,15}$ and included a constant 


\section{Box 1. Inclusion criteria for interview study}

- A diagnosis of chronic obstructive pulmonary disease (COPD)

- Aged $>40$ years

- A forced expiratory volume in 1 second (FEV1) post-bronchodilator $<80 \%$ AND predicted ratio of FEV1 to forced vital capacity (FVC) $<0.70 \mathrm{OR}$ clinical decision of suitability for patients who are unable to provide a spirometry reading (on clinical grounds) at full assessment. The patient must have prior clinical evidence of COPD, that is, obstructive spirometry within the last 10 years; radiological evidence of emphysema.

- Smoking history $>10$ pack years.

- Medical Research Council dyspnoea scale $\geq 2$.

- Registered with a general practice and with an exacerbation of COPD requiring home treatment or hospital admission in the previous year, or referred for pulmonary rehabilitation.

- Absence of other significant lung disease.

- Absence of chronic heart failure defined by the New York Heart Association classification system as severe (Grade IV).

- Able to give informed consent.

- Life expectancy $>3$ months

- Able to adequately understand written and verbal English.

comparative method; open, axial, and selective coding; and memo writing, to identify theoretical links and concepts from the data

To ensure rigour of the coding process, there were reviews of transcripts and identified themes, and a random sample of interviews (15\%) were double coded by a senior qualitative researcher external to the project. Both of these processes aided transparency and credibility of the findings.

\section{RESULTS}

\section{Patient characteristics}

Twenty-three patients took part in the pilot study and, of these, 19 took part in the interview study. Four patients were not included in the interview study as the

\section{Table 1. Patient baseline characteristics}

\section{Characteristic}

\begin{tabular}{lc}
\hline Sex, $n$ & 11 \\
Male & 8 \\
Female & 67 (50-85) \\
\hline Mean age (range), years & 6 \\
\hline Severity of COPD, $n$ & 10 \\
GOLD stage II & 3 \\
GOLD stage III & \\
GOLD stage IV & 4 \\
\hline Duration of symptoms, years 1 to $\geq 20$ & 15 \\
\hline Home oxygen use, $n$ & 11 \\
\hline Previous attendance of pulmonary rehabilitation programme, $n$ & 8 \\
\hline Living set up, $n$ & \\
Living with spouse/family & \\
Living alone & \\
\hline GOLD = Global Initiative for Chronic Obstructive Lung Disease classification (http://mww.goldcopd.org/). ${ }^{16}$
\end{tabular}

interviewer was not able to schedule an interview prior to receiving the mHealth intervention. The baseline characteristics of patients are given in Table 1. All 19 patients were interviewed before the intervention, but only 15 patients were interviewed after the intervention because of drop out $(n=2)$, death $(n=1)$, and unavailability $(n=1)$.

\section{Qualitative findings}

The process of double coding by an external researcher as well as discussions of the coding process within the research team did not present any discrepancies in the findings.

The main themes emerging from the interview data were:

- patients' transition from being uncertain about their ability to use the technology to being confident to use it;

- the way in which the mHealth intervention addressed patient concerns about fragmented care by offering a sense of continuity of care and, thereby, reassurance;

- increased patient awareness of the variability of symptoms; and

- the way the mHealth intervention appeared to support patients' selfmanagement behaviour.

From uncertainty of ability to use technology to 'ease of use'. Patients had a varied previous experience in using computers and mobile phone technology, ranging from none to everyday use of smart phones and tablet computers. Unsurprisingly, those who had little or no previous experience in using computers were more likely to express concerns over using the mHealth application:

'First of all when, because I kept saying, "I'm never going to cope" I didn't really want to do it after I said I would. Only because my sister said, "Go on, you can do it". But I am not, I've never been on these things or whatever. (PR034)

Interestingly, the perceived inability to cope' with using the mHealth application did not only relate to their lack of experience but also to age:

'I said, "no, I wouldn't cope with something like that. I'm too old to bother".' (PR034)

'But [uh] it's a job when you're old, you get, you know the family says, "Oh, have a laptop, 
have a ..." [breathless] / get muddled up with my television, don't, remote controls so Yes, I am quite happy to be where I am. I can't be bothered with new stuff.' (HA004)

Yet despite these initial concerns, patients in this study were able to use the mHealth application regardless of their previous experience. In addition, even those who initially expressed concern over their age reported during the second interview that they were able to use the mHealth application effectively:

So I know exactly what to do with that. So I'm quite good. I'm quite pleased with myself.'(PR034)

Well, I was all right ... I was all right. (HA004)

It appeared that despite initial concerns about both age and ability to use the mHealth application effectively, none of the patients in this study had significant problems in using the application.

From 'disruption of care' to 'reassurance and perceived continuity of care'. Patients expressed satisfaction with their curren care but indicated a sense of disrupted care in terms of their contact with healthcare professionals. This was not necessarily attributed to the behaviour of professionals but more to their own reluctance in making contact with them. These perceptions appeared to relate to patients not wanting to be 'burdensome' and 'bothering' nurses, who were perceived to be busy, resulting in feelings of 'being left out there':

I don't like to bother them [nurses] to be honest, you know if I can cope.' (HA007)

"'Oh we'll come and see you such and such." And they never come ... I mean I know they're busy, don't get me wrong, I do know they're busy, but I just feel a bit like, left out there.' (HA001)

Another reason for patients to avoid seeking contact with healthcare professionals was the potential of unnecessary visits, and therefore these were often delayed:

I'm not a great one for to-ing and fro-ing to the doctor unnecessarily.' (HA006)

During the second interview, a sense of reassurance and continuity of care through mHealth monitoring was identified. Patients were aware that data were not monitored by the research nurse on a daily basis and were advised (both in written form and verbally prior to taking written consent) to contact healthcare services in the usual way in an emergency. Yet the virtual link offered by the mHealth intervention appeared to reassure patients and gave a sense of continuity of care:

'It's nice to know that you've got it ... you're being monitored you know, and it gives a sense, you know, this is all getting back to some central computer and it's all being monitored. So somebody's actually looking at how you are, without me actually going to the doctors, so you get that feeling that you're being looked after.' (HA014)

The main aspect that underpinned this sense of continuity appeared to relate to the sharing of patients' self-monitoring data with the research nurse, even though this was infrequent and did not replace current care:

I think having the system that will monitor it, and somebody'll have a record. '(HA015)

'You're being looked after really, looked over and there is someone who is looking at your condition ... I did like the idea that someone would be looking over me.' (HA002)

Increased awareness of variability of symptoms. Patients also indicated an increased awareness of the variability of their symptoms. Although they acknowledged the often unpredictable nature of their COPD-related symptoms during the first interview, during the second interview they emphasised a much more structured approach to reviewing their condition and how they felt not just over a number of days but also within a 24-hour period. As patients were answering questions about their symptoms as well as monitoring their oxygen saturation on a daily basis, they felt encouraged to think more about how they were feeling each day and throughout the day:

It makes me think about the problem more during the day ... you know you're sort of analysing yourself how you're feeling." (HA007)

'It makes you stop and think about ... how things are.' (HA0O2)

This appeared particularly beneficial as patients reported they would often ignore 
their symptoms or be less aware of a symptom change, as explained during the second interview:

I think it's, it's probably just a good thing to be aware because I might start not bothering to become aware, if I wasn't now that I'm not using it [mHealth intervention].' (HA002)

I am the sort of person who ignores my own, whatever it is l've had that are too nasty things. I tend to ignore it. [um] And I don't think, "How am I feeling today?". The only time I thought about how I'm feeling today was when I filled it in. "How have I felt today?" And then I would assess how I felt today.' (HA006)

As part of the mHealth application, patients were asked to monitor their oxygen saturations on a daily basis, and initially patients were uncertain how to interpret oxygen levels:

I'm not quite sure what it should be. [er] Yes, I don't know what's bad. Say it went down to 80 ... I mean I'd probably be dead by then, but I don't know. You see I've no idea.' (HA013)

However, after having used the application for 6 months, they appeared to develop an understanding of their individual 'normal' levels, despite not receiving any additional information or training about oximetry and normal oxygen saturation levels:

'When I am feeling alright it's [oxygen saturation] about 90.' (PR034)

'It seems to vary between 94 and 97.' (HA011)

Although not all patients paid the same amount of attention to their oxygen saturation levels, there was a sense of 'wanting to know' oxygen saturation levels as this would aid their decision-making process when feeling unwell:

'Definitely l'd want to know because then I might be able to do something about it.' (HA012)

The patients in this interview study welcomed the self-monitoring of oxygen saturation levels and perceived this as beneficial in managing their COPD.

Supporting self-management behaviour. As well as using the oxygen saturation data provided by the mHealth application as an aid to self-manage COPD, patients also perceived the tablet computer, as a whole, as supporting their self-management behaviour. It reminded patients of the need to engage in self-management:

'It's a presence in the home, it encourages me to do what I call breathing exercises. (HA006)

It also reinforced routines that included adherence to regular medication:

'Because, I knew l'd got to do that ... do my puffers and then I go and have my shower and then I do my thing. I'd got it all in, and I think it was because of that that I'd got to do, that I done it so regular. And took my tablets as well.' (PR034)

Patients expressed the view that using the mHealth application was beneficial to their self-management, not just in terms of monitoring and adherence to medication, but also engaging with mood issues. Patients expressed feelings of low mood but explained that using the symptom diary (which included a general question of wellbeing) made them realise they were in a better frame of mind than they initially thought:

'It's a good thing to be aware [of COPD changes] and become aware ... and also if you're feeling a bit down ... and you got to say how you feel, you stop and think and think I don't have to feel down and then you think "I feel good". '(HA0O2)

Yet some patients perceived the mHealth application as less useful. It is interesting to note that those who 'felt it was a waste of time, '(PR032) appeared to be less engaged with managing their COPD, and stated that:

They [respiratory nurses] tell me to do this when I'm not well and they [respiratory nurses] come and see me,' (HA001).

Thus they appeared to rely more on healthcare professionals to make decisions about treating exacerbations and often suffered from comorbidities that impacted equally if not more on their health. Other characteristics, such as COPD severity, length of diagnosis or familiarity with communication technologies appeared to be less strongly linked to level of engagement with the mHealth intervention.

\section{DISCUSSION}

\section{Summary}

The mHealth intervention overall was 
viewed positively by patients. After a period of use, it was seen to be easy to use, providing reassurance and a sense of continuity of care, and supporting patients self-management behaviour. A small number of patients ( $n=2)$ questioned these benefits, although this group appeared less engaged in self-management behaviour from the outset than those who felt positive about the mHealth application. Patients had been made aware that their symptom and pulse oximetry data were not reviewed on a daily basis. Yet despite this, patients found the application easy to use and the virtual link created by sending self-monitoring data to a research nurse provided patients with a sense of continuity of care, as well as reassurance of 'being looked after'. Having used the mHealth application regularly for 6 months, patients felt more aware of the variability of their symptoms and more encouraged to engage in self-management behaviour.

\section{Strengths and limitations}

Published research shows that the benefits of telehealth, as assessed by randomised controlled trials, are still inconclusive.? Qualitative studies have been carried out to explore the patient experience of using telehealth but these seem to have included patients with a variety of comorbidities, static telehealth equipment, ${ }^{10}$ and limited data collection to a one-off interview post-intervention or mid-intervention. ${ }^{11}$ This current study explored the patient experience within one condition (COPD), using a mobile telehealth application. A mobile device may offer advantages over static equipment, such as the ability to move the equipment to fit with everyday life, take it outside the home, and accept it as an everyday technological device rather than merely equipment associated with illhealth. In addition, a longitudinal approach was used, interviewing patients pre- and post-intervention.

Since this study used a qualitative methodology, the aim was not to seek statistical generalisability but rather theoretical transferability by using purposive sampling to capture a wide range of patient characteristics and their experience. ${ }^{17} \mathrm{~A}$ qualitative enquiry as part of a larger telehealth trial can enrich the sometimes limited picture provided by quantitative data and thus provide valuable insight into how patients engage with the technology. ${ }^{18}$ The use of longitudinal, individual in-depth interviews pre- and post-intervention allowed patients to voice their perceptions and experience of current care and perceived impact of a mHealth application. The findings presented here add to our understanding of how a telehealth application can support selfmanagement for patients with COPD.

There were some limitations of this study. The patient sample was recruited within two NHS trusts within the same region and the care provided for patients with COPD may differ in other NHS regions. Patients were also recruited during early winter and data collection was completed by early summer thus limiting the exploration of factors related to seasonal variance. The views and perceptions of healthcare professionals involved in the care of patients with COPD in the community were not sought, an aspect which the authors aim to address in subsequent research. The qualitative researcher was part of the EDGE COPD team, and therefore it is possible that preconceptions of the intervention may have influenced data collection and analysis. However, as with this study, an experienced researcher uses wellestablished methodological approaches Ireflexivity, double-coding, and discussion of analytic process with the principal investigator) to minimise such bias.

\section{Comparison with existing literature}

Findings from this study strongly suggest that patients found the application easy to use regardless of their age or previous computer experience. This is an important finding since some authors suggest that the use of tablet computers may be problematic for older people, ${ }^{19}$ and patients in other qualitative studies have voiced concern over the need for technical competence to use telehealth applications. ${ }^{10}$ The lack of elicited negative comments about usability in this study may therefore reflect the success of an intervention development process in which the focus was to provide a simple, intuitive application that could be used by people regardless of their previous computer experience.

The findings of this study show that patients perceived the telehealth application as providing reassurance and a sense of continuity of care, which is supported by other qualitative studies exploring telehealth/care for patients with COPD. ${ }^{11,20}$ One study reported patient perceptions of threats to identity, independence, and selfcare. ${ }^{10}$ Yet this study related to the Whole System Demonstrator trial, with interview findings from a patient sample with highly varied care contexts, ranging from young disabled people to older patients requiring palliative care. Thus their care needs 
and the way these patients engaged with the care provided differed and may have had an impact on how they engaged with the telehealth intervention. In addition, the telehealth equipment described in the Whole System Demonstrator trial was static, which led to some patients associating the equipment with being 'stuck indoors', whereas the telehealth application used in this study was mobile and could be taken anywhere.

The findings presented here showed an increased awareness of variability of symptoms, particularly in relation to onset of exacerbations and recovery. This allowed patients to make a more informed decision about their self-management, when to contact healthcare professionals, and to monitor periods of deterioration and recovery, a finding that is also supported by previous research. 8.9 Yet other research has shown that self-monitoring posed additional stress for patients who felt that monitoring of clinical parameters, such as oxygen saturation, was the role of a nurse and they did not want to be burdened with this task. ${ }^{10}$ Some authors have found that telemonitoring worried some patients if they felt unprepared to interpret clinical data, ${ }^{9}$ an issue that was not identified in this study. Yet some participants found the use of the mHealth application less beneficial than others, and these participants appeared to also be less engaged in self-management behaviour. This suggests that the extent to which patients engage successfully in selfmanagement as supported by telehealth applications, in particular the selfmonitoring of symptoms and clinical signs, may be related to other health behaviours. This emphasises that telehealth appears to have a supporting role in self-management rather than replacing current care, and that patients' engagement in self-management is necessary to achieve successful engagement with a telehealth application.

\section{Implications for practice and research}

This study suggests that mHealth applications can be developed so they are easy to use, even for those with little or no previous computer experience, and such telehealth interventions have the potential to benefit patients in terms of perceived continuity of care and supporting self-management behaviour. However, it appears crucial that any telehealth application complements rather than replaces current care, and thus supports and encourages self-management behaviour. Yet, telehealth applications that require patients to engage in selfmanagement behaviour rather than merely provide self-monitoring data may not be perceived as beneficial by all patients, as these interventions require patients to actively engage with managing their long-term condition. Findings from this study suggest that patients are able to recognise norms and patterns of their oxygen saturation data with minimal training. However, the perceived benefits of mHealth monitoring to support selfmanagement could be further improved by assessing patients' knowledge about clinical parameters, health behaviours, and self-management approach when implementing such interventions.

Future research is needed to explore how this mHealth intervention is used over a longer period that includes seasonal variation. In addition, it will be important to explore how patients perceive the individual components of this mHealth intervention, such as monitoring of clinical data, multimedia content of the patient education module, and symptom diaries.

\section{Provenance}

Freely submitted; externally peer reviewed.

\section{Competing interests}

The authors have declared no competing interests.

\section{Acknowledgements}

The authors thank all members of the EDGE COPD research team: Dr Christy Toms, Dr Louise Jones, Heather Rutter, Professor Carl Heneghan, Dr Oliver Gibson, Dr Carmelo Velardo, Dr Syed Shah, Dr Gari Glifford, Dr Andreas Triantafyllidis and the patients who took part in this study.

\section{Discuss this article}

Contribute and read comments about this article: www. bjgp.org.uk/letters 


\section{REFERENCES}

1. Yohannes AM, Roomi J, Waters K, Connolly MJ. Quality of life in elderly patients with COPD: measurement and predictive factors. Respir Med 1998; 92: $1231-1236$.

2. Pinnock $H$, Kendall M, Murray SA, et al. Living and dying with severe chronic obstructive pulmonary disease: multi-perspective longitudinal qualitative study. BMJ 2011; 342: d142.

3. National Institute for Health and Clinical Excellence. Chronic obstructive pulmonary disease. Costing report. London: NICE, 2011

4. Taylor SJ, Candy B, Bryar RM, et al. Effectiveness of innovations in nurse led chronic disease management for patients with chronic obstructive pulmonary disease: systematic review of evidence. BMJ 2005; 331: 485.

5. Effing T, Monninkhof EM, van der Valk PD, et al. Self-management education for patients with chronic obstructive pulmonary disease. In: Cochrane Collaboration. The Cochrane Library. Issue 4. Oxford: Update Software, 2007.

6. Kielmann T, Huby G, Powell A, et al. From support to boundary: a qualitative study of the border between self-care and professional care. Patient Educ Couns 2010; 79(1): 55-61.

7. McLean S, Nurmatov U, Liu JL, et al. Telehealthcare for chronic obstructive pulmonary disease. In: Cochrane Collaboration. The Cochrane Library. Issue 7. Oxford: Update Software, 2011

8. Ure J, Pinnock H, Hanley J, et al. Piloting tele-monitoring in COPD: a mixed methods exploration of issues in design and implementation. Prim Care Respir J 2012; 21(1): 57-64.

9. Huniche L, Dinesen B, Nielsen C, et al. Patients' use of self-monitored readings for managing everyday life with COPD: a qualitative study. Telemed J E Health 2013; 19(5): 396-402

10. Sanders C, Rogers A, Bowen R, et al. Exploring barriers to participation and adoption of telehealth and telecare within the Whole System Demonstrator trial: a qualitative study. BMC Health Serv Res 2012; 12: 220.

11. Fairbrother P, Pinnock H, Hanley J, et al. Continuity, but at what cost? The impact of telemonitoring COPD on continuities of care: a qualitative study. Prim Care Respir J 2012; 21(3): 322-328

12. Farmer $A$, Toms $C$, Hardinge $M$, et al. Self-management support using an internet-linked tablet computer (the EDGE platform) based intervention in chronic obstructive pulmonary disease: protocol for the EDGE-COPD randomized controlled trial. BMJ Open 2014; 4: e004437. DOI:10.1136/ bmjopen-2013-004437.

13. Bucknall CE, Miller G, Lloyd SM, et al. Glasgow supported self-management trial (GSUST) for patients with moderate to severe COPD: randomised controlled trial. BMJ 2012; 344: e1060.

14. Charmaz K. 'Discovering' chronic illness: using grounded theory. Soc Sci Med 1990; 30(11): 1161-1172.

15. Charmaz K. Constructing grounded theory: a practical guide through qualitative analysis. London: SAGE, 2006.

16. Global Initiative for Chronic Obstructive Lung Disease. Global strategy for the diagnosis, management and prevention of COPD. http://www.goldcopd.org/ guidelines-global-strategy-for-diagnosis-management.html laccessed 8 May 2014]

17. Coyne IT. Sampling in qualitative research. Purposeful and theoretical sampling; merging or clear boundaries? J Adv Nurs 1997; 26(3): 623-630.

18. Cafazzo JA, Seto E, Jadad AR. Qualitative studies enrich telemonitoring research, practice, and technology design. Prim Care Respir J 2012; 21(1): 10-11.

19. Hess R, Santucci A, McTigue $K$, et al. Patient difficulty using tablet computers to screen in primary care. J Gen Intern Med 2008; 23(4): 476-480.

20. Horton K. The use of telecare for people with chronic obstructive pulmonary disease: implications for management. J Nurs Manag 2008: 16(2): 173-180. 


\section{Appendix 1. Interview 1 questions}

As you know we are currently developing a mobile health system to support people living with COPD to manage their own condition. We would like to find out a bit more about how you currently experience your condition, and how you manage COPD at home at the moment.

1. First of all, could you tell me about how your condition affects you on a daily basis?

2. How do you currently manage your disease on a good day (self, carer, healthcare professional input)?

3. Tell me a bit more about what a bad day is like.

4. Do you know what an exacerbation is?

5. How do your symptoms change when you feel an exacerbation coming on? How does it start?

6. How do you manage your condition when you have a bad day?

7. What are the main issues that you face in managing your condition?

8. How well do you feel you understand your condition?

9. How confident do you feel in managing your COPD at home?

10. What is your experience of the current care provided to you by the respiratory team (nurses/GPs/ pulmonary rehabilitation/clinic)?

11. Is there anything you can think of that might facilitate the management of your condition?

12. How do you feel about using IT in your daily life (mobile phones, computer, internet, smart phones)?

13. How do you feel about using technology to help manage your condition?

14. How do you think a system, such as the handheld computer which you will receive, might be beneficial to you in managing your COPD on a daily basis?

15. Can you think of any difficulties/challenges such a system might bring?

16. What do you expect from this application in terms of managing your condition?

Many thanks for taking part in this research.

\section{Appendix 2. Interview 2 questions}

1. How have you got on in using the handheld computer over the last 6 months?

2. How has using the mHealth system affected the way you manage your disease?

3. In what ways has the mHealth system affected your everyday life?

4. What were the main benefits in using the system?

5. What were the main issues/difficulties you were facing when using the system?

6. What would you like to see changed about how you use the system?

7. How could we improve the system?

8. Anything else?

Many thanks for taking part in this research. 